\title{
Issues in quantification of registered respiratory gated PET/CT in the lung
}

\author{
Vesna Cuplov ${ }^{1,2, *}$, Beverley F. Holman ${ }^{1}$, Jamie McClelland ${ }^{3}$, \\ Marc Modat $^{4}$, Brian F. Hutton ${ }^{1}$ and Kris Thielemans ${ }^{1}$ \\ ${ }^{1}$ Institute of Nuclear Medicine, UCLH, 235 Euston Road, London NW1 2BU, UK \\ ${ }^{2}$ IMIV, U1023 Inserm/CEA/Université Paris-Sud and ERL 9218 CNRS, Université \\ Paris-Saclay, CEA/SHFJ, Orsay, France \\ ${ }^{3}$ Centre for Medical Image Computing, Department of Medical Physics and \\ Biomedical Engineering, University College London, London WC1E 6BT, UK \\ ${ }^{4}$ Translational Imaging Group, Centre for Medical Image Computing, University \\ College London, London WC1E 6BT, UK
}

E-mail: vesna.cuplov@gmail.com

September 2017

\begin{abstract}
PET/CT quantification of lung tissue is limited by several difficulties: the lung density and local volume changes during respiration, the anatomical mismatch between PET and CT and the relative contributions of tissue, air and blood to the PET signal (the tissue fraction effect). Air Fraction Correction (AFC) has been shown to improve PET image quantification in the lungs. Methods to correct for the movement and anatomical mismatch involve respiratory gating and image registration techniques. While conventional registration methods only account for spatial mismatch, the Jacobian determinant of the deformable registration transformation field can be used to estimate local volume changes and could therefore potentially be used to correct (i.e. Jacobian Correction, JC) the PET signal for changes in concentration due to local volume changes. This work aims to investigate the relationship between variations in the lung due to respiration, specifically density, tracer concentration and local volume changes. In particular, we study the effect of AFC and JC on PET quantitation after registration of respiratory gated PET/CT patient data. Six patients suffering from lung cancer with solitary pulmonary nodules underwent ${ }^{18} \mathrm{~F}$-FDG PET/cine$\mathrm{CT}$. The PET data were gated into six respiratory gates using displacement gating based on a Real-time Position Management (RPM) signal and reconstructed with matched gated CT. The PET tracer concentration and tissue density were extracted from registered gated PET and CT images before and after corrections (AFC or JC) and compared to the values from the reference images. Before correction, we observed a linear correlation between the PET tracer concentration values and density. Across all gates and patients, the maximum relative change in PET tracer concentration before (after) AFC was found to be $16.2 \%(4.1 \%)$ and the maximum relative change in tissue density and PET tracer concentration before (after) JC was found to be $17.1 \%$ (5.5\%) and $16.2 \%(6.8 \%)$ respectively. Overall our results show that both AFC or JC largely explain the observed changes in PET tracer activity over the respiratory cycle. We also speculate that a second order effect is related to change in fluid content but this needs further investigation. Consequently, either AFC or JC is recommended when combining lung PET images from different gates to reduce noise.
\end{abstract}


PACS numbers: 87.57.nj, 87.57.Q-, 87.57.uk

Keywords: pulmonary PET/CT, respiration, density, registration, local volume changes, tissue fraction effect

\section{Introduction}

The interest of using PET/CT imaging as a procedure for investigating pulmonary diseases caused by infection, inflammation or fibrosis has recently increased (Groves et al. 2009, Inoue et al. 2009, Win et al. 2012, Win et al. 2014, Abdulla et al. 2014, Scherer and Chen 2016). PET/CT imaging could play an important role in identifying lung abnormalities and assessing disease progression and treatment response in pulmonary diseases. Lung quantification in $\mathrm{PET} / \mathrm{CT}$ is challenging due to the presence of large fractions of air (Lambrou et al. 2011), blood (Holman et al. 2015), and potentially other extracellular fluids (Chen et al. 2017) and respiratory motion. Lung inflation influences the overall lung density, whereas gravity causes lung regional differences in density by increasing the blood component and decreasing alveolar expansion (Verschakelen et al. 1993). Therefore, PET and CT signals are expected to vary between respiratory stages, adding to the complexity of PET/CT image quantification in the lung.

The effects of lung expansion (respiration) on tissue density has been widely studied on CT images (Verschakelen et al. 1993, Simon 2000). Most studies use deformable image registration between images from a 4DCT dataset representing different phases of the respiratory cycle (usually end-inhalation and end-exhalation) and compute various properties of the registered images and the obtained transformation fields. Lung deformation, which corresponds to a measurement of the lung local volume change (expansion or contraction), is determined from the determinant of the Jacobian (the matrix of the first-order partial derivatives) of the lung CT registration deformation field (Reinhardt et al. 2008). Christensen et al. have shown a correlation between tracking lung motion using image registration and spirometry data in lung cancer patients (Christensen et al. 2007). Reinhardt et al. compared the degree of regional lung expansion (measured using the Jacobian of the registration displacement field of respiratory gated CT images) to ventilation measures acquired using xenon CT imaging in five sheep (Reinhardt et al. 2008). The Jacobian determinant distribution changes at different stages of the breathing cycle (Ding 2008, Amelon et al. 2011). Observed density changes in the lung during respiration are correlated with local volume changes and the Jacobian determinant (Jahani et al. 2014). These studies have shown that local lung density changes are related to local volume changes (as estimated by image registration). Guerrero et al. reported that the total tissue mass changes up to $10 \%$ over the respiratory cycle (Guerrero et al. 2006). During respiration, fluids (such as blood and lymph) could move in and out of the lungs (El-Chemaly et al. 2008), therefore the change in tissue mass in the lungs could be attributed to changes in the amount of 
fluid in the lungs.

The influence of respiration on quantification in PET has been well studied for oncological and cardiological applications. However, the effect in pulmonary studies has been less investigated, although the impact is likely to be large. In the lung, tracer concentration is sensitive to the amount of air present. Correcting PET images for the air component is known as the air fraction correction (AFC) which can be done based on the CT (Lambrou et al. 2011). After AFC, the PET values are proportional to amount of tracer per gram. Respiration will cause movement, but also air fraction and density changes, and the expansion will affect tracer concentration. A lung voxel generally contains parenchymal (i.e. alveolar), airway wall, vascular wall (e.g. endothelial cells) and immune cells, as well as blood, water (i.e. extracellular fluid) and air (Chen et al. 2017). Its density will depend on the amount of cells, fluids and air that it contains, whereas the signal measured by PET will correspond to the amount of tracer in all the different voxel components and their relative fractions (Holman et al. 2015, Chen et al. 2017). Changes in tracer concentration and their relation to changes in density due to respiration are therefore non-trivial and have not yet been investigated to the best of our knowledge.

To reduce the blurring and the associated increase in partial volume effect caused by the respiratory movement, PET data can be gated (Nehmeh et al. 2002, Vines et al. 2007). To achieve accurate PET quantitation, PET and CT need to match at each gate to ensure correct attenuation correction (AC). Using an accurate estimate of the density for $\mathrm{AC}$ has been shown to be crucial when studying the lung (Holman et al. 2016). However, gating leads to increased noise in the gated PET images. Several gates can be combined with registration, either during or after image reconstruction, to generate one single motion corrected PET image (Nehmeh and Erdi 2008, Rahmim et al. 2013). To avoid the increased dose to the patient associated with gated CT, several authors deform a single CT to match the PET gates, for instance based on registering non-attenuation corrected (NAC) PET images (Kalantari and Wang 2017, Wells et al. 2010) or during reconstruction (Bousse et al. 2016, Rezaei et al. 2016). However, conventional registration methods follow anatomical tissue deformation and account for spatial mismatch, but do not correct for density and concentration changes associated with expansion. Mass-preserving registration methods do take the local volume change into account, usually via the Jacobian determinant (Yin et al. 2009, Thielemans et al. 2009, Gigengack et al. 2012) or alternatively via explicit calculation (Ue et al. 2006), with only the latter reference applied to lung tracer concentrations, albeit in SPECT. However, as noted above, it is not a priori clear if it is desirable or required to correct PET images for local volume changes during lung expansion. Correcting PET/CT data for local volume change during lung expansion is known as Jacobian correction (JC).

In this paper, we study how to combine lung respiratory gated PET/CT images using registration and preserve lung density and PET tracer concentration by applying AFC or JC. To avoid difficulties with registration of PET images and the effect observed on PET quantitation if the wrong CTAC is used (Holman et al. 2016), we use 
patient data with cine-CT (acquired at free breathing) gated to match the PET data. We investigate the relationship between density changes measured in CT, tracer concentration changes measured in PET and local volume changes obtained from the Jacobian of the registration deformation field applied on gated CT matched to gated PET.

\section{Theory}

\subsection{Air Fraction Correction using gated PET/gated CT}

The interpretation of PET/CT images for lung disease is affected by the tissue fraction effect (TFE). Lambrou et al. originally introduced the concept that observed activity concentration in the lung is affected by the fraction of air present, requiring appropriate correction (Lambrou et al. 2011). The voxel fractional air volume $\left(V_{a}\right)$ is obtained from the CT images :

$$
V_{a}=\frac{H U_{\text {voxel }}-H U_{\text {tissue }}}{H U_{\text {air }}-H U_{\text {tissue }}}
$$

where $H U$ is the $\mathrm{CT}$ image voxel Hounsfield Units, $H U_{\text {tissue }}=45$ and $H U_{\text {air }}=-1000$. In our lung study, the lung mask is density based. Therefore, within the mask, the CT voxel $H U$ will mostly be lower than the $H U$ of the tissue (i.e. 45). Negative values of the air fraction are set to zero. The voxel fractional tissue volume, which can be assumed to be proportional to the density of tissue in the voxel, is given by :

$$
V_{t}=1-V_{a} .
$$

To account for the air content in the lung voxel, the PET tracer Standardized Uptake Value (SUV) after AFC is :

$$
S U V_{A F C}=\frac{S U V_{\text {measured }}}{V_{t}}
$$

where $S U V_{\text {measured }}$ is the PET tracer concentration measured directly from the PET image. The PET voxel values after AFC are those of the tissue (without the air component).

\subsection{Registration of gated PET/gated CT and Local Volume Change}

During breathing, lung density varies due to lung volume change. Correcting for local volume change should account for these variations due to changes in the air content of the lung. The local volume change can be extracted from the Jacobian determinant $(|J|)$ of the spatial transformation which can be estimated by image registration (i.e. registration to a reference image) and is defined as the following ratio :

$$
|J|=\frac{\text { deformed } \text { voxel volume }}{\text { reference voxel volume }}
$$

To account for the local volume change that occurs during respiration, registered (floating image registered to a reference) fractional air volume $\left(V_{a}\right)$ and PET images 
can be Jacobian corrected (JC) :

$$
\text { Image }{ }_{J C}^{\text {floating } \rightarrow \text { reference }}=\frac{\text { Image }^{\text {floating } \rightarrow \text { reference }}}{|J|^{\text {floating } \rightarrow \text { reference }}} \quad \text { Image }=\left\{V_{a}, \mathrm{PET}\right\}
$$

The registered fractional air volume and PET voxel values after JC are expected to be more similar to those of the reference image.

\section{Methods}

\subsection{Patient Data Acquisition}

Data used for this retrospective study were from 6 patients suffering from lung cancer with solitary pulmonary nodules who underwent ${ }^{18}$ F-FDG PET/cine-CT using a GE Discovery STE PET/CT scanner (Mawlawi et al. 2004) with list mode enabled. With the exception of the small nodules (diameter less than $4 \mathrm{~cm}$ in all patients), the patients used in this study were all considered to have normal lung tissue. Patients were scanned approximately 1 hour after injection according to the normal clinical protocol. Patients breathing was monitored using an external device, the Varian Realtime Position Management (RPM) system which measures the vertical position of a block placed on the patient chest near the diaphragm. Cine-CT data was collected with free breathing for approximately one breathing cycle over each of $10 \mathrm{CT}$ bed positions, each covering an axial Field of View (FOV) of $2 \mathrm{~cm}$. The X-ray source full rotation period was set to 0.5 seconds, its voltage to $140 \mathrm{kVp}$ and current to $90 \mathrm{~mA}$. The time between cine-CT images was set to 0.45 seconds. A single PET bed position (axial FOV $15.4 \mathrm{~cm}$ ) was acquired over 6-8 minutes. Patients were positioned to have the primary nodules in the centre of the axial FOV.

\subsection{Patient Analysis}

The original PET list mode data, the cine-CT DICOM data together with the RPM PET and CT gating signals were processed with the GE PET toolbox, a proprietary suite of MATLAB (The MathWorks, Inc., Natick, Massachusetts, USA) and C tools for gating and image reconstruction. The RPM signal was used to split the PET and CT data into 6 different respiratory states using displacement gating, where the range of the PET and CT signals was determined manually to cover the full extent of the breathing cycles, i.e. ignoring peaks related to irregular breathing. Gate 1 corresponds to the end-inhalation breathing stage and gate 6 to the end-exhalation breathing stage. A full scan reconstruction was applied to the gated CT $(512 \times 512 \times 80$ voxels of size $0.9766 \times 0.9766 \times 2.5 \mathrm{~mm}^{3}$ ). Gated PET data were reconstructed with the standard settings used on the GE Discovery scanner console $(192 \times 192 \times 47$ voxels of size $3.64 \times 3.64 \times 3.27 \mathrm{~mm}^{3}$ ) with OSEM (Hudson and Larkin 1994), both without and

with the attenuation correction maps derived from the corresponding gated CT. After reconstruction, a visual check was performed comparing the non-attenuation corrected PET images with the gated CT images to confirm that PET and CT gates matched. 
3.2.1. Creating the Lung Masks Lungs were segmented from the gated CT images with an in-house tool based on the Insight Segmentation and Registration Toolkit (ITK) (Johnson et al. 2016). In a previous step, the bed was removed from the CT images. The resulting image (CTnobed) consisted of the patient body and a background set to -200. The segmentation consisted in performing a thresholding of the end-exhalation CTnobed image, depending on whether the corresponding image voxel values lie between the two thresholds in HU [-200, 3000]. These bounds cover most of the human body HU except air and those organs containing air. Everything within these bounds is set to 0, and the rest (i.e. objects with a considerable amount of air: lungs, esophagus, trachea, bowel gas) to 1 . This resulted in a tissue mask with islands corresponding to lowdensity regions. These islands were identified with a connected component analysis and objects smaller than $0.5 \%$ of the CT image volume were removed. The resulting binary lung mask was resampled to the PET image size using a nearest neighbour interpolation technique. Unfortunately due to the limited size of the PET axial FOV and the position of the patients, lungs were not entirely in the FOV. To avoid lung edges and spurious effects due to the significant presence of the liver and heart uptake, the final lung region of interest LROI was obtained by erosion using a binary morphological transformation with a ball structuring element (Nikopoulos and Pitas 2000) with a 3-dimensional radius of 4 pixels (i.e. $14.56 \times 14.56 \times 13.08 \mathrm{~mm}^{3}$ ). For all patients (except patient 1 ), 20 slices were removed from the bottom of the LROI image and 4 slices from the top in order to accommodate for the movement (the lung mask stays in the FOV during inhalation) and to exclude noisy PET data in the end-planes. For patient 1, 15 slices were removed from the bottom of the LROI image and 6 slices from the top. Figure 1 shows an example of an eroded and cropped lung mask obtained by segmenting the reference gated CT image of patient 1 (i.e. exhalation).
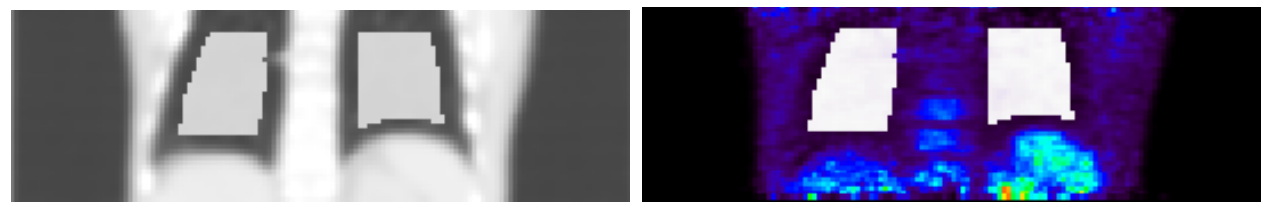

Figure 1. Example of one patient LROI overlaid on the CT (left) and PET (right) images corresponding to an exhalation stage of the breathing cycle. The lung mask was eroded to avoid lung edges and obvious artefacts (liver, heart uptake). All images and masks are resampled to the PET space.

3.2.2. Registration Each gated CT image was registered to a reference CT image, chosen to be the exhalation gate (i.e. smallest lung volume), using an affine followed by a non-linear registration method as implemented in NiftyReg (Ourselin et al. 2001, Modat et al. 2010). The lung masks were such that only the intensities within the lungs are considered to optimise the transformations and all other areas are ignored. The affine registration utilises a block matching approach, where the normalised cross-correlation is used as a measure of similarity to align the most similar blocks. A least trimmed 
squared approach is used to extract a robust affine transformation from the block correspondences. To further improve the robustness of the registration a symmetric implementation was used (Modat et al. 2014). The obtained affine transformations are then used to initialise the subsequent non-linear registration step avoiding the need to resample the input images several times. A stationary velocity field parametrised by a cubic B-Spline control point grid is used to model the non-linear deformation (Modat et al. 2012). The control point grid is discretised with one control point every 10 voxels along each axis. Similarly to the affine scheme, a symmetric implementation is used to maximise robustness and remove any bias towards the directionality of the registration. To promote a smooth transformation, the bending energy is used as a constraint. The locally normalised cross-correlation (Cachier et al. 2003) is used as a measure of similarity with a Gaussian kernel standard deviation set to 5 voxels along each axis. The use of local versions of the normalised cross-correlation renders the measure of similarity robust to local intensity variation induced by the ventilation process during the respiratory cycle.

3.2.3. Obtaining the Fractional Tissue Volume Images The CT scan dataset was used to derive fractional tissue volume images on a voxel by voxel basis using Eq. 2. The CT images were processed to approximately match the PET resolution by using a resampling technique. Smoothing with a Gaussian filter has also been applied. This filter convolves the image with a 3D Gaussian kernel using a full width at half maximum (FWHM) set to $8.48 \mathrm{~mm}$.

3.2.4. Analysis We performed two types of analysis which are illustrated in Figure 2. In the Moved Images analysis, the gated images were transformed to match the reference gate using the deformation fields derived from the gated CT. This analysis was used to investigate the change in PET and CT mean ROI values (after registration) without corrections and with either JC or AFC corrections. In contrast, in the Moved Masks analysis, the lung mask of the reference image was deformed (i.e. deformed-LROI or dLROI) to be able to extract total tissue volume and total tracer activity for the same part of the lung in every gate.

- Moved Images analysis : For each gate, PET and CT images are resampled to the reference position using the CT-derived deformation field. The voxel fractional tissue volume and PET tracer SUV are extracted from the deformed CT and PET images using the reference image lung mask (i.e. LROI in Section 3.2.1). This analysis will be used to determine from the LROI a mean value of the fractional tissue volume $\left(V_{t}^{L R O I}\right)$ and the PET tracer SUV $\left(S U V^{L R O I}\right)$.

- Moved Masks analysis : For each gate, the reference position LROI is resampled using the inverse of the CT-derived deformation, such that the resampled mask matches the lung position for that gate. The lung total tissue volume $(\mathcal{V})$ and total PET tracer activity normalised with the injected activity and patient weight $(\mathcal{A})$ 


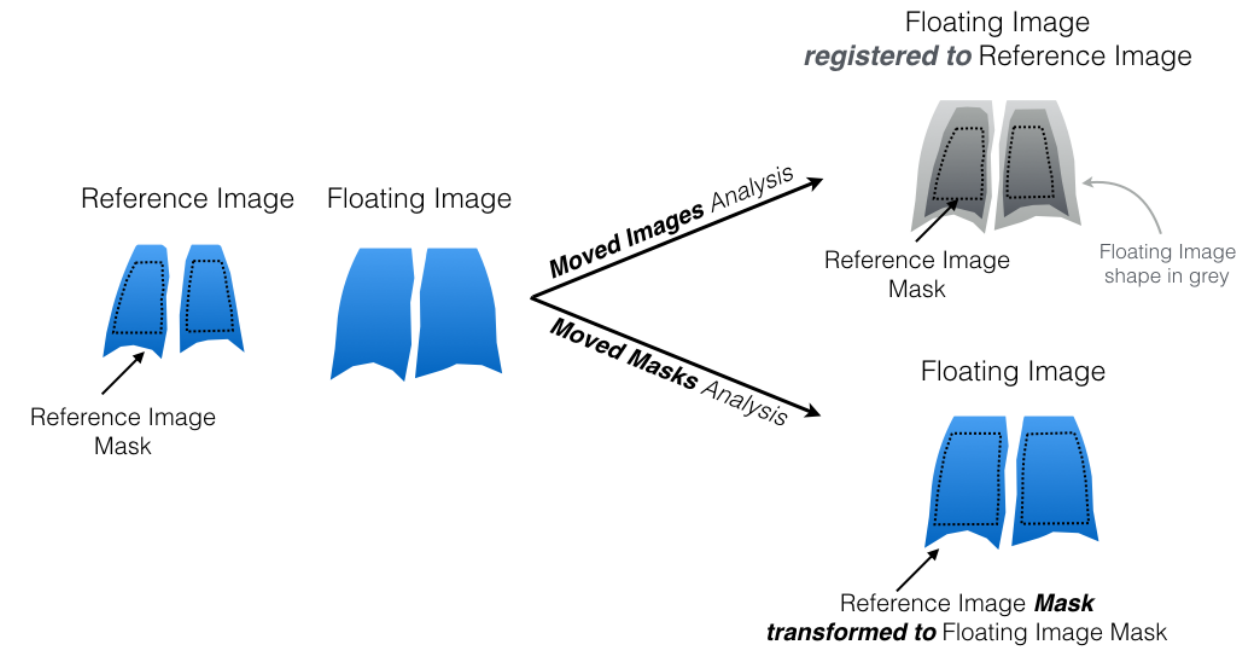

Figure 2. In the Moved Images analysis, the floating image was transformed to match the reference image. In the Moved Masks analysis, the lung mask of the reference image was deformed to match the same region of the lung in the floating image. Both reference and floating images are shown with the lung not entirely in the FOV. Lung masks are eroded and cropped. For the Moved Images analysis, the shape of the floating image is shown in light grey and the floating image after registration to the reference image is shown in dark grey.

contained in the dLROI are extracted from un-deformed gated CT and PET images using the transformed mask (i.e. dLROI) :

$$
\begin{aligned}
& \mathcal{V}=V_{t}^{d L R O I} \times v_{d L R O I} \\
& \mathcal{A}=S U V^{d L R O I} \times v_{d L R O I}
\end{aligned}
$$

where $v_{d L R O I}$ corresponds to the volume of the deformed-LROI.

The relative change (in \%) in fractional tissue volume $\left(\delta V_{t}^{L R O I}\right)$ and PET tracer SUV $\left(\delta S U V^{L R O I}\right)$ between registered gates and the reference gate (relative to the reference gate) are obtained following the Moved Images analysis :

$$
\begin{aligned}
& \delta V_{t}^{L R O I}=\frac{V_{t}^{L R O I, \text { gate }}-V_{t}^{\text {LROI,reference }}}{V_{t}^{\text {LROI,reference }}} \times 100 \\
& \delta S U V^{L R O I}=\frac{S U V^{L R O I, \text { gate }}-S U V^{L R O I, \text { reference }}}{S U V^{L R O I, \text { reference }}} \times 100
\end{aligned}
$$

The relative change (in \%) in lung total tissue volume $(\delta \mathcal{V})$ and total PET tracer activity $(\delta \mathcal{A})$ between registered gates and the reference gate (relative to the reference gate) are computed similar to Eqs. 6 and 7. The measurement of these relative changes (before and after correction: AFC or JC) will provide an estimate of the importance for a density correction of registered respiratory gated PET/CT images. 


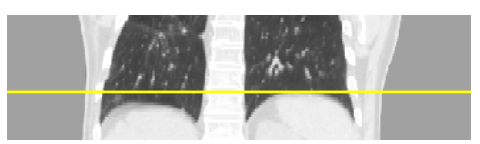

Reference Image

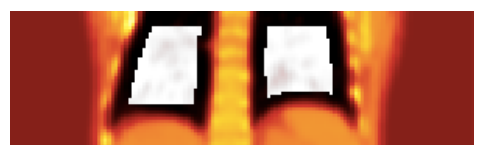

$f_{\text {tissue }}=0.1563$
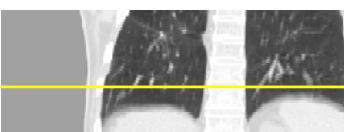

Floating Image

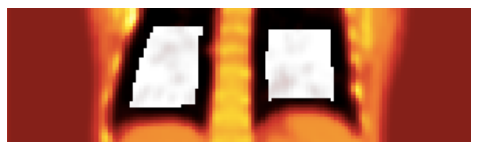

$f_{\text {tissue }}=0.1577$
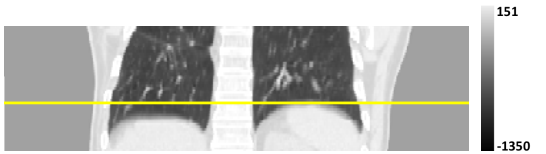

Registered Image

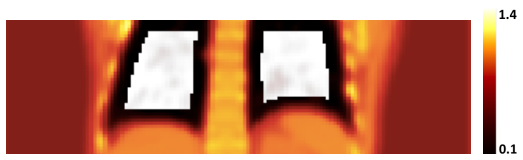

$f_{\text {tissue }}=0.1526$

Figure 3. Top row shows an example of one patient CT registration: gated CT in the reference (end of exhalation) gate (left); gated (gate 2) CT (middle) and registration of gate 2 to the reference (right). The yellow line shows the position of the bottom of patient's left lung (displayed on the right of the image). The bottom row shows the corresponding fractional tissue volume images. The reference and registered images are overlaid with the reference image lung mask. The floating image shows the moved mask (reference lung mask resampled using the inverse of the CT-derived deformation, such that it matches the lung position for the floating gate). These masks were used to extract the fractional tissue volume values quoted below.

\subsection{Air fraction correction}

Registered gated PET images were corrected for air fraction using the voxel fractional air volume maps extracted from the gated CT images (see Eq. 3). Using the Moved Images analysis, the relative change in the PET tracer SUV $\left(\delta S U V^{L R O I}\right)$ was extracted from registered gated PET images (before and after AFC) following Eq. 7.

\subsection{Jacobian correction}

The Jacobian determinant maps were obtained from the deformation fields (6 gates, 5 deformation fields) of the registration of gated CT images to the reference image (i.e. exhalation). Following the Moved Images analysis, the relative change in fractional tissue volume $\left(\delta V_{t}^{L R O I}\right)$ and PET tracer SUV $\left(\delta S U V^{L R O I}\right)$ were calculated before and after Jacobian correction (local volume change correction) using Eqs. 6 and 7.

\section{Results}

\subsection{Patient Analysis}

Figure 3 shows an example of one patient CT registration. The registration was visually judged to be of very good quality. Figure 4 shows an example of one patient Jacobian determinant map obtained after the registration of two gated CT images.

Figure 5 plots the fractional tissue volume (i.e. representative of tissue density) and

the PET tracer concentration after registration (without any corrections) normalised to the value in the reference (i.e. gate 6 corresponds to exhalation). For both gated CT and PET, image intensities after resampling (using the registration deformation field) 


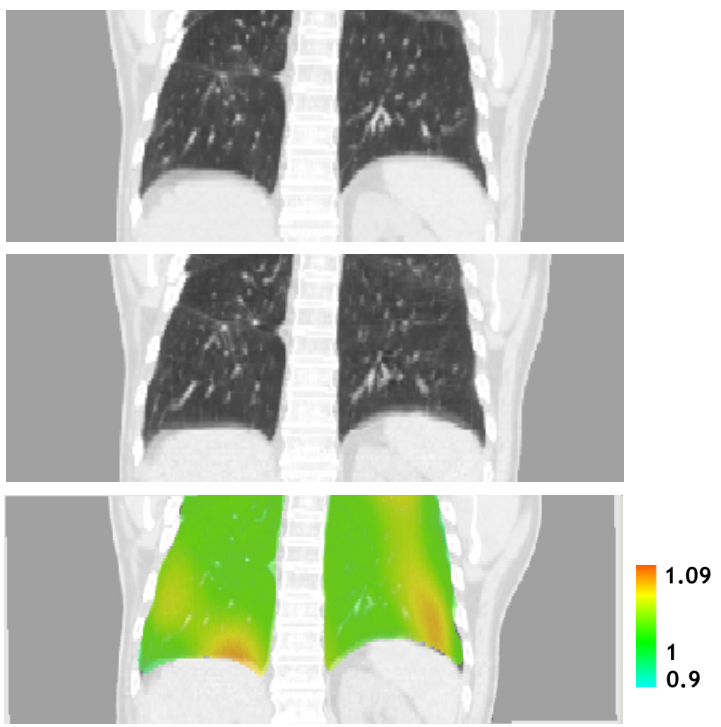

Figure 4. Example of one patient $\mathrm{CT}$ registration with the corresponding Jacobian determinant map: gated $\mathrm{CT}$ in the reference gate (end-expiration) (top); gated $\mathrm{CT}$ in the floating gate (end-inhalation) (middle) and registered image overlaid with the Jacobian determinant map (bottom).

are lower than at exhalation. Across all gates and patients, the maximum (mean)
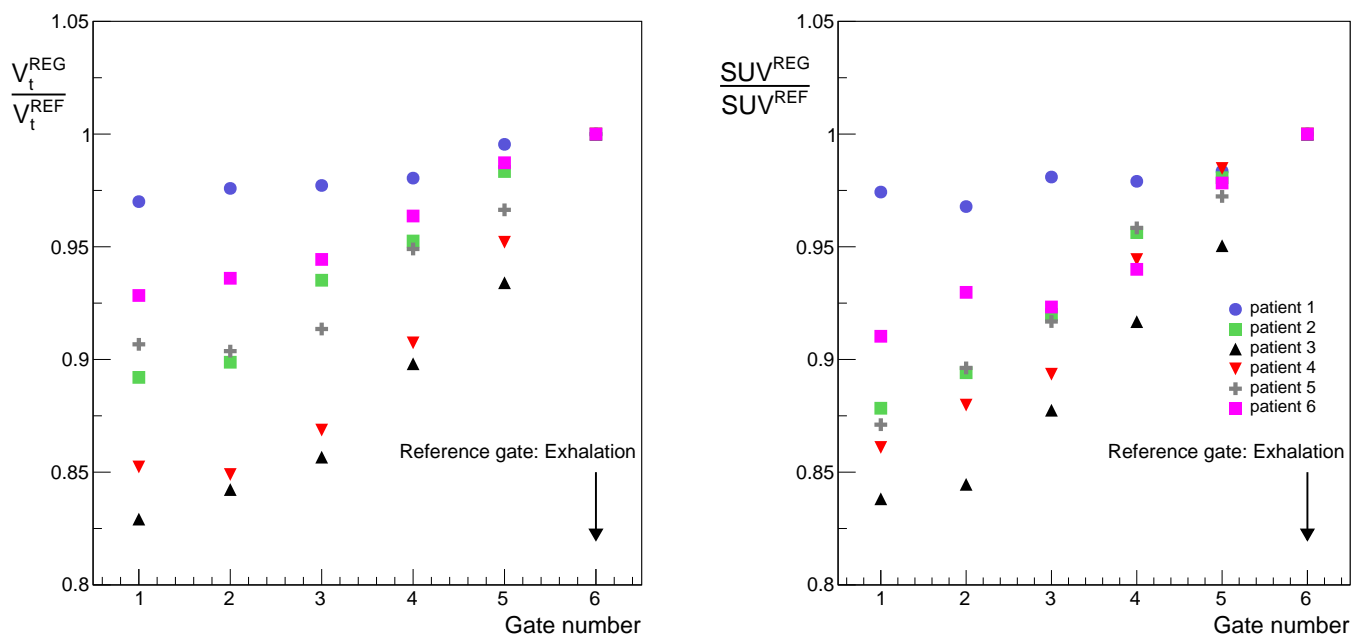

Figure 5. Fractional tissue volume and PET tracer concentration (using the Moved Images analysis) after registration and normalised to the value in the reference for each gate before corrections.

relative change in fractional tissue volume $\left(\delta V_{t}^{L R O I}\right)$ and PET tracer SUV $\left(\delta S U V^{L R O I}\right)$ between registered gates and the reference gate (relative to the reference gate) without any correction is $17.1 \%(7.5 \%)$ and $16.2 \%$ (7.3\%) respectively (see also Figure 8 ). 


\subsection{Accounting for density changes: air fraction correction}

Figure 6 shows the relationship between the fractional tissue volume and the PET tracer concentration after registration (before and after air fraction correction) normalised to the value in the reference. After AFC, the registered PET signal is relatively stable (i.e. gate independent). Figure 7 shows the maximum relative change in PET tracer
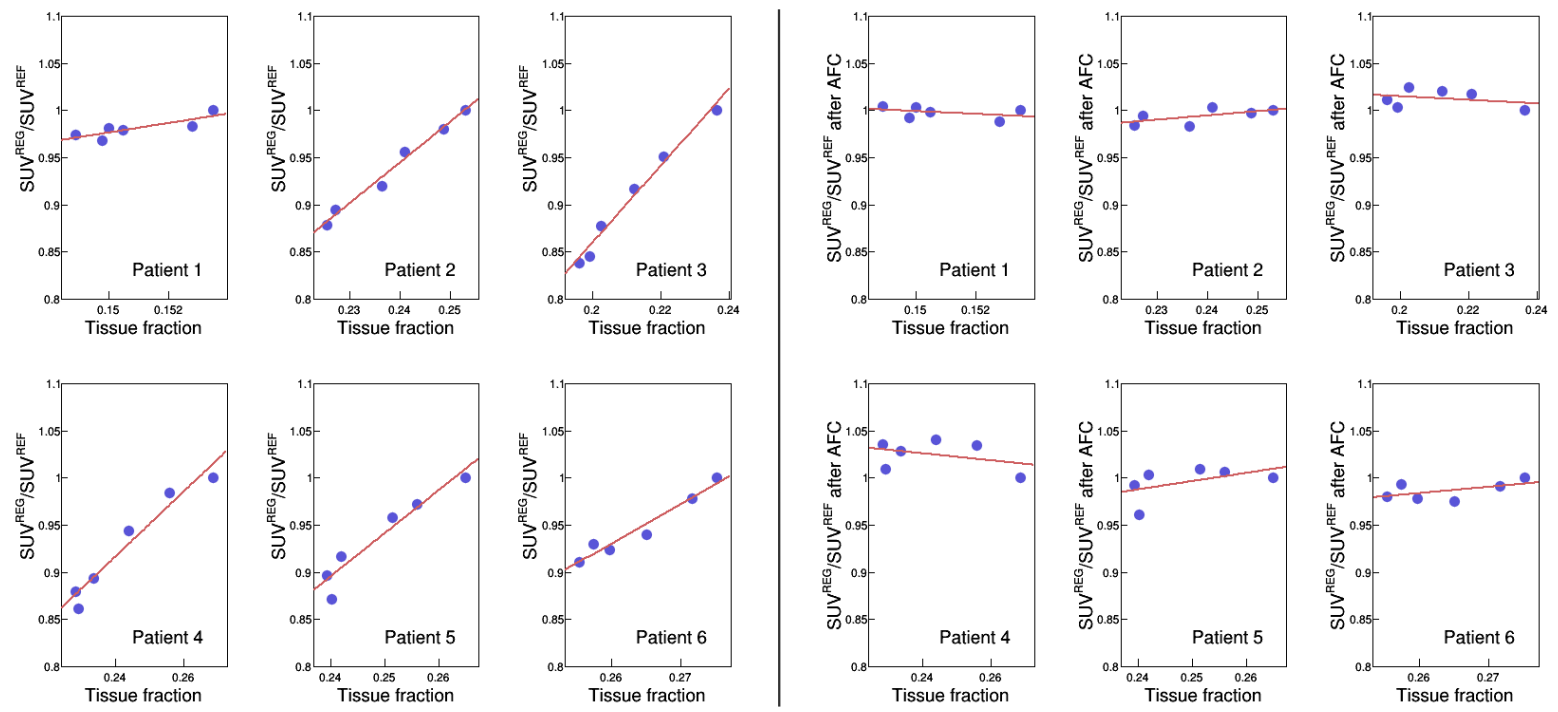

Before air fraction correction

After air fraction correction

Figure 6. PET tracer concentration after registration and normalised to the value in the reference as a function of the fractional tissue volume in each gate for each patient: (left) before and (right) after air fraction correction and using the Moved Images analysis.

SUV (Max. $\delta S U V^{L R O I}$ ) before and after air fraction correction. Across all gates and patients, the mean relative change in PET tracer SUV before (after) AFC is $6.1 \% \pm$ $5.0 \%(1.2 \% \pm 1.2 \%)$ and the maximum relative difference before (after) AFC is $16.2 \%$ $(4.1 \%)$.

\subsection{Accounting for local volume changes: Jacobian correction}

Figure 8 displays the maximum relative change in fractional tissue volume (Max. $\delta V_{t}^{L R O I}$ ) and PET tracer SUV (Max. $\delta S U V^{L R O I}$ ) between registered gates and the reference, before and after Jacobian correction using the Moved Images analysis. Across all gates and patients, the maximum relative change in fractional tissue volume and PET tracer SUV before (after) Jacobian correction is $17.1 \%(5.5 \%)$ and $16.2 \%(6.8 \%)$ respectively. The mean relative change in fractional tissue volume and PET tracer SUV before (after) Jacobian correction is $6.3 \pm 5.2(1.8 \pm 1.8)$ and $6.1 \pm 5.0(1.8 \pm 1.8)$ respectively. 


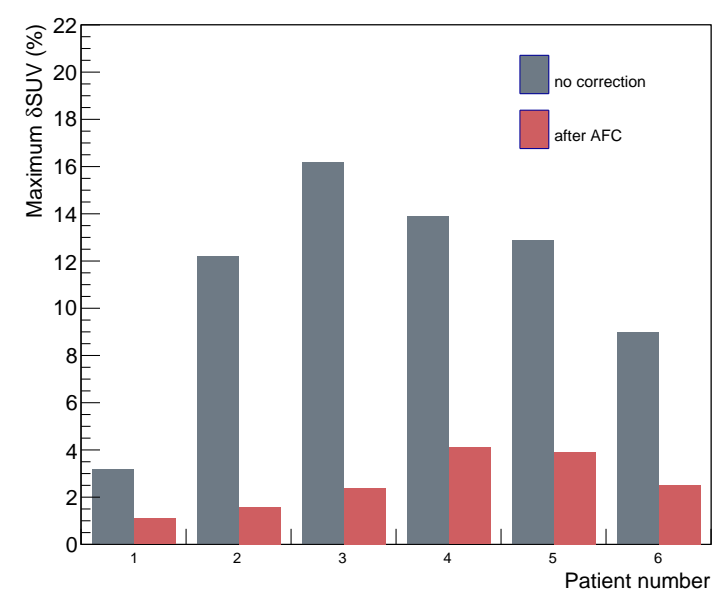

Figure 7. Maximum relative change in PET tracer SUV before and after air fraction correction following the Moved Images analysis.
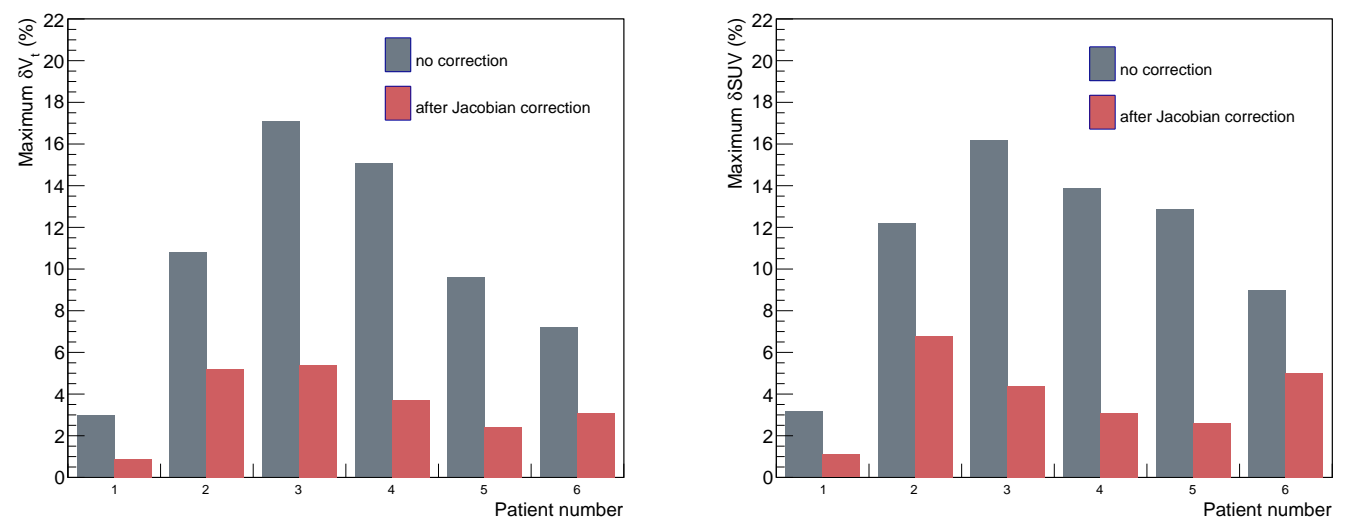

Figure 8. Maximum relative change in fractional tissue volume and PET tracer SUV before and after Jacobian correction for all patients following the Moved Images analysis.

\subsection{Relative change in total Tissue Volume and Activity}

The results in the previous sections are largely consistent with the mass-preservation hypothesis. To test this further, Figure 9 shows the relative change in total tissue volume $(\delta \mathcal{V})$ as a function of the relative change in total PET tracer activity $(\delta \mathcal{A})$. No corrections were applied and the lung total tissue volume and total PET tracer activity were determined using the Moved Masks analysis. If mass-preservation would hold, we would expect $\delta \mathcal{V}$ and $\delta \mathcal{A}$ to be independent of the gate. Across all gates and patients, the maximum (mean) relative change in total tissue volume and total PET tracer activity is $-6.7 \%(2.1 \%)$ and $-6.5 \%(2 \%)$ respectively (see Figure 9). For patients 1,4 and 5 , the changes in total tissue volume and total activity are very small (maximum 1.3 

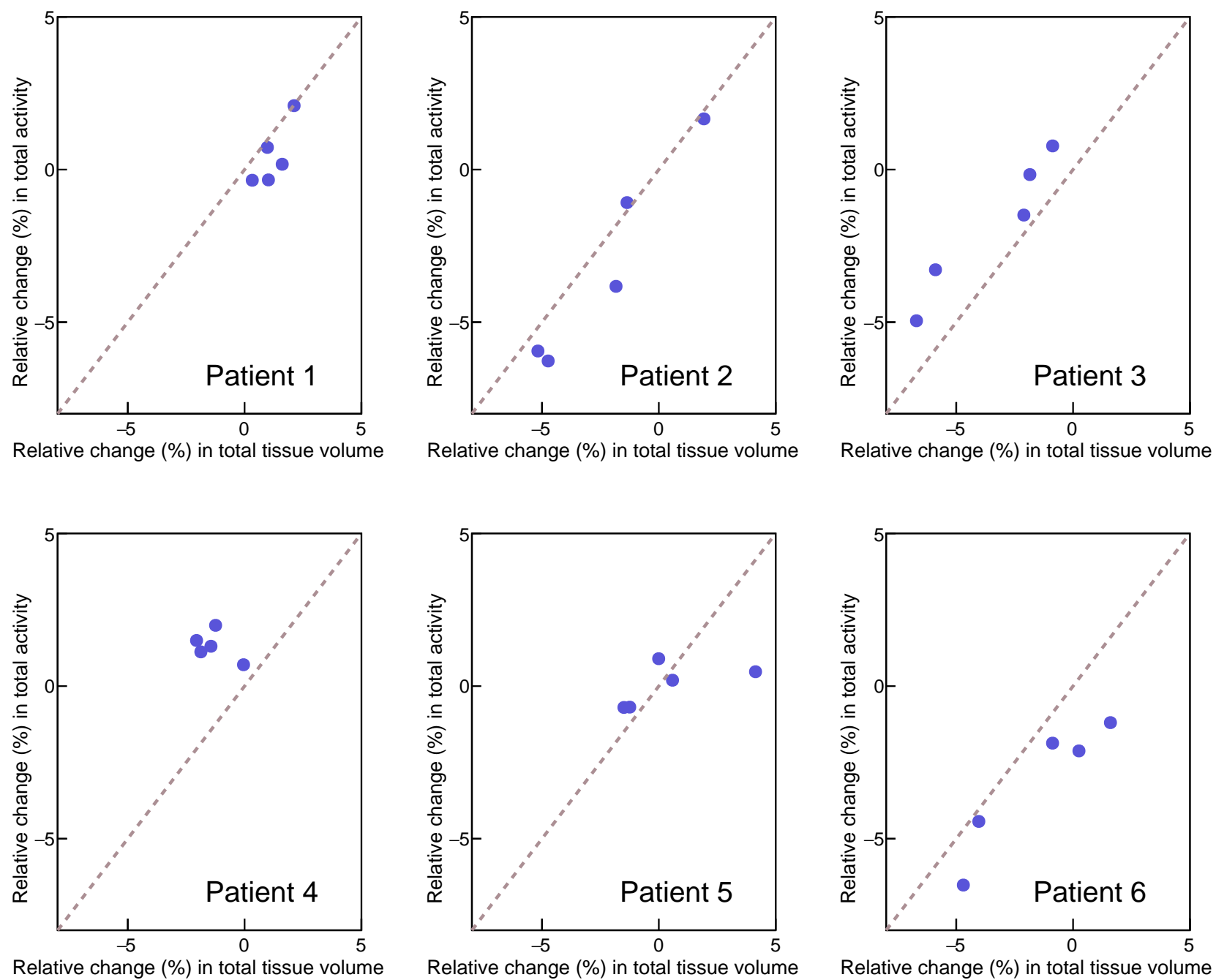

Figure 9. Relative change in total tissue volume as a function of the relative change in total PET tracer activity, without any corrections.

$\%$ and $0.9 \%$ respectively) as expected. However, for patients 2,3 and 6 , the changes are $6 \%$ with a linear correlation between $\delta \mathcal{V}$ and $\delta \mathcal{A}$, suggesting that some substance containing PET tracer entered (or left) the region.

\section{Discussion}

The aim of this work is to investigate the extent of changes over the respiratory cycle in thoracic image data. While a few papers have suggested to use mass-preserving registration, most of the existing literature on respiratory motion correction for PET considers only deformation and interpolates the images to a different gate. This paper therefore investigates how the image values change between respiratory gates.

With reference to Figure (5), without any correction, the maximum relative change (between the registered and the reference image) in fractional tissue volume and PET 
tracer concentration is $17.1 \%$ and $16.2 \%$ respectively, implying that these quantities are not conserved after the image registration process. The registration aligns images, but the voxel intensities in the registered image should become similar to the intensities in the reference. A correction is needed in order to account for volume/density changes. After applying the AFC, the registered PET signal appears to be gate independent (Figure 6). As illustrated in Figure 7, the maximum change in PET tracer concentration after AFC reduces to $4.1 \%$. This substantial reduction can be explained by the fact that $\mathrm{AFC}$ aims to correct the registered PET signal intensities to account for differences in density.

When using the Jacobian determinant map, obtained from the registration deformation field, to correct CT and PET images for local volume changes, it has been found that the maximum relative change in fractional tissue volume and PET tracer concentration after JC is $5.5 \%$ and $6.8 \%$ respectively (Figure 8 ).

These results show that to a large extent, changes (due to respiration) in lung tissue density, air fraction, PET tracer concentration and local volume changes (derived from the Jacobian determinant of the registration deformation field) are all proportional and non-negligible.

Some residual changes in the corrected fractional tissue volume and PET tracer concentration were observed. This is consistent with the results of the Moved Image analysis. The maximum relative change in total tissue volume and total PET tracer activity in the lungs is $-6.7 \%$ and $-6.5 \%$ respectively (Figure 9). For patients 2, 3 and 6, a linear correlation is observed between these total quantities, suggesting that some substance containing PET tracer entered (or left) the region. For other patients the observed changes are too small to draw any conclusions. These results could be explained by a component of the lung tissue (other than parenchyma) that flows in and out of the lungs over the breathing cycle, such as blood or lymph. The lymphatic circulation is a vital component in lung biology (El-Chemaly et al. 2008). Blood is a large component of the lung voxel $(\sim 20 \%)$ (Wollmer et al. 1984) and adds to the PET signal (Holman et al. 2015). Brudin et al. have explored the relationships between the volume elements within the voxel (gas, blood and tissue) and ventilation (Brudin et al. 1994, Rhodes and Hughes 1995). They have shown that there was an inverse correlation between regional vascular and alveolar gas volumes. This implies that vascular volume is inversely related to alveolar expansion, which in itself is a determinant of regional ventilation. Measuring changes in the blood fraction over the respiratory cycle, e.g. by using gated ${ }^{11} \mathrm{CO}$ data, will help to understand this further but will be the subject of future work.

This study is limited by the small number of patient datasets. In addition, the use of the RPM for gating can still lead to remaining mismatches between PET and CT, especially during irregular breathing (Sun and Mok 2012). In this study, no coaching was used for controlling the breathing. A further limitation of this work is that results were aggregated over a large lung ROI. Results for smaller ROIs would have been dependent on several elements such as the ROI position, the registration accuracy and the gating 
of the cine-CT data due to irregular breathing among patients.

$\mathrm{PET} /$ cine-CT is not generally available in the clinic, while essential for this study. Therefore data from a previous oncology study looking at solitary pulmonary nodules was utilised to investigate the lung tissue. With the exception of the small nodules (diameter less than $4 \mathrm{~cm}$ in all patients), the patients used in this study were all considered to have normal lung tissue. The nodules themselves were not considered in this paper. Due to their small size reliable computation of the Jacobian determinant in the nodules could not be performed and the work by Holman et al. suggested that tumour quantitation was not affected by density effects anyway. The relations between density, air fraction, tracer concentration and local volume changes will need further investigation for patients with both oncological and diffuse lung diseases.

When combining (using a registration technique) respiratory gated PET images to reduce noise, our work has shown that applying a correction is necessary for quantitation in order to take into account the changes in the lung due to respiration. AFC of PET images was recommended (Lambrou et al. 2011, Holman et al. 2015) to be able to measure the amount of tracer per tissue, independent of the amount of air in the region. The results of this paper show that the PET tracer concentration corrected for the air fraction is indeed (largely) independent of respiration. Therefore, if matched respiratory gated $\mathrm{CT}$ data are available (i.e. CT acquisition in cine mode), AFC of gated PET images followed by registration and combination is a viable approach. If only an inspiration/expiration CT pair is available due to dose considerations, data at intermediate stages could be obtained by interpolation (Mok et al. 2013) combined with the Jacobian correction to correct for density changes. If only a single CT scan (i.e. snapshot of the respiratory cycle) is available, any CT image representing a different stage of the breathing cycle could theoretically be generated by transforming the single CT using the deformation field obtained from the registration of gated PET data. The Jacobian determinant of the registration field should then be used to correct both the obtained CT images and the reconstructed PET images for local volume changes during respiration. These suggestions will be evaluated in future work. Compared to using static CT at several stages of the respiratory cycle, the acquisition in cine mode is a great asset in testing the method at any stage of the respiratory cycle. The approximate radiation dose (CT-Expo, Stamm and Nagel 2002) from the cine-CT $(2.3 \mathrm{mSv})$ is significantly higher than the low dose CT $(0.3 \mathrm{mSv})$. Nevertheless this work used cine-CT to demonstrate the need for correcting for variation lung density between respiratory stages. Methods using lower dose CT acquisitions will also be investigated hoping that it will lead to extension of methods that avoid cine-CT to increase their accuracy.

\section{Conclusion}

Respiration affects PET/CT quantitation in the lung. These variations seem dominated by local volume changes. To a large extent, Air Fraction Correction (AFC) or Jacobian 
Correction (JC) explains the change in lung density and PET tracer activity over the respiratory cycle. These corrections are needed when using registration techniques to combine gated images.

\section{Acknowledgments}

We acknowledge funding support from GlaxoSmithKline (STU100028576) and the Engineering and Physical Sciences Research Council (EP/K0052781/1). Also, this project is supported by researchers at the National Institute for Health Research, University College London Hospitals Biomedical Research Centre. The authors would like to thank General Electric Healthcare for use of their software. The authors would also like to thank Valentino Bettinardi, Maria Carla Gilardi, and Ferruccio Fazio from Hospedale San Raffaele, HSR Milano (Italy) for providing patient data.

\section{References}

Abdulla S, Salavati A, Saboury B, Basu S, Torigian D and Alavi A 2014, "Quantitative assessment of global lung inflammation following radiation therapy using FDG PET/CT: a pilot study," Eur. J. Nucl. Med. Mol. Imaging 41 350-6.

Amelon R, Cao K, Ding K, Christensen G E, Reinhardt J M and Raghavan M L 2011, "Threedimensional characterization of regional lung deformation," J. Biomech. 44(13) 2489-95.

Bousse A, Bertolli O, Atkinson D, Arridge S, Ourselin S, Hutton B F and Thielemans K 2016, "Maximum-likelihood joint image reconstruction and motion estimation with misaligned attenuation in TOF-PET/CT," Phys. Med. Biol. 61 11-19.

Brudin L H, Rhodes C G, Valind S O, Jones T and Hughes J M 1994 , "Interrelationships between regional blood flow, blood volume, and ventilation in supine humans," J. Appl. Physiol. 76 1205-10.

Cachier P, Bardinet E, Dormont D, Pennec X and Ayache N 2003, "Iconic feature based nonrigid registration: the PASHA algorithm," Computer Vision and Image Understanding 89(2-3) 272-98.

Chen D L, Cheriyan J, Chilvers E, Choudoury G, Coello C, Connell M, Fisk M, Groves A M, Gunn R N, Holman B F, Hutton B F, Lee S, MacNee W, Mohan D, Parr D, Subramanian D, TalSinger R, Thielemans K, van Beek E JR, Vass L, Wellen J W, Wilkinson I and Wilson F J 2017 , "Quantification of Lung PET Images: Challenges and Opportunities," J. Nucl. Med. 58(2) 201-7.

Christensen G E, Song J H, Lu W, Naqa I E, Low D A 2007, "Tracking lung tissue motion and expansion/compression with inverse consistent image registration and spirometry," Med. Phys. 34(6) 2155-63.

CT-Expo tool - http://www.sascrad.com.

Ding K 2008, "Registration-based regional lung mechanical analysis," Master's thesis, University of Iowa.

El-Chemaly S, Levine S and Moss J 2008, "Lymphatics in Lung Disease," Annals of the New York Academy of Sciences 1131 195-202.

Gigengack F, Ruthotto L, Burger M, Wolters C H, Jiang X, and Schäfers K P 2012, "Motion correction in dual gated cardiac PET using mass-preserving image registration," IEEE transactions on medical imaging 31(3) 698-712.

Groves A M, Win T, Screaton N J, Berovic M, Endozo R, Booth H, Kayani I, Menezes L J, Dickson J C and Ell P J 2009, "Idiopathic pulmonary fibrosis and diffuse parenchymal lung disease: implications from initial experience with 18f-FDG PET/CT," J. Nucl. Med. 50(4) 538-45.

Guerrero T, Sanders K, Castillo E, Zhang Y, Bidaut L, Pan T and Komaki R 2006, "Dynamic ventilation imaging from four-dimensional computed tomography," Phys Med Biol. 51 777-91. 
Holman B F, Cuplov V, Millner L, Hutton B, Maher T, Groves A and Thielemans K 2015, "Improved correction for the tissue fraction effect in lung PET/CT imaging," Phys Med Biol. 60(18) 7387-402.

Holman B F, Cuplov V, Hutton B F, Groves A M and Thielemans K 2016, "The effect of respiratory induced density variations on non-TOF PET quantitation in the lung," Phys Med Biol. 61(8) $3148-63$.

Hudson M and Larkin R 1994, "Accelerated image reconstruction using ordered subsets of projection data," IEEE Trans. Med. Imag 13 601-9.

Inoue K, Okada K, Taki Y, Goto R, Kinomura S and Fukuda H 2009, "18FDG uptake associated with CT density on PET/CT in lungs with and without chronic interstitial lung diseases," Ann. Nucl. Med. 23(3) 277-81.

Johnson H J, McCormick M M, Ibáñez L and the Insight Software Consortium 2016, https://itk.org/ItkSoftwareGuide.pdf.

Jahani N, Yin Y, Hoffman E and Lin C-L 2014, "Assessment of regional non-linear tissue deformation and air volume change of human lungs via image registration," J. Biomech. 47 1626-33.

Kalantari F and Wang J 2017, "Attenuation correction in 4D-PET using a single-phase attenuation map and rigidity-adaptive deformable registration," Med. Phys. 44(2) 522-32.

Lambrou T, Groves A M, Erlandsson K, Screaton N, Endozo R, Win T, Porter J C and Hutton B F 2011, "The importance of correction for tissue fraction effects in lung pet: preliminary findings," Eur. J. Nucl. Med. Mol. Imaging 38(12) 2238-46.

Mawlawi O, Podoloff D A, Kohlmyer S, Williams J J, Stearns C W, Culp R F and Macapinlac H 2004, "Performance characteristics of a newly developed PET/CT scanner using NEMA standards in 2D and 3D modes," Journal of Nuclear Medicine 45(10) 1734-42.

Modat M, Ridgway G R, Taylor Z A, Lehmann M, Barnes J, Fox N C, Hawkes D J and Ourselin S 2010, "Fast free-form deformation using graphics processing units," Comput Meth Prog Bio. 98(3) $278-84$.

Modat M, Ridgway G R, Daga P, Cardoso M J and Ourselin S 2012, "Parametric non-rigid registration using a stationary velocity field," IEEE Workshop on Mathematical Methods in Biomedical Image Analysis (MMBIA) http://doi.org/10.1109/MMBIA.2012.6164745.

Modat M, Cash D M, Daga P, Winston G P, Duncan J S and Ourselin S 2014, "Global image registration using a symmetric block-matching approach," Journal of Medical Imaging 1(2) 024003.

Mok G S, Sun T, Huang T C and Vai M I 2013, "Interpolated average CT for attenuation correction in PET-a simulation study," IEEE Trans Biomed Eng 60(7) 1927-34.

Nehmeh S A and Erdi Y E 2008, "Respiratory motion in positron emission tomography/computed tomography: A review," Seminars in Nuclear Medicine 38(3) 167-76.

Nehmeh S A, Erdi Y E, Ling C C, Rosenzweig K E, Schoder H, Larson S M, Macapinlac H A, Squire O D and Humm J L 2002, "Effect of Respiratory Gating on Quantifying PET Images of Lung Cancer ," J. Nucl. Med. 43(7) 876-81.

Nikopoulos N and Pitas I 2000, "An efficient algorithm for 3d binary morphological transformations with $3 \mathrm{~d}$ structuring elements for arbitrary size and shape," IEEE Transactions on Image Processing 9(3) 283-6.

Ourselin S, Roche A, Subsol G, Pennec X and Ayache N 2001, "Reconstructing a 3d structure from serial histological sections," Image and Vision Computing 19(1-2) 25-31.

Rahmim A, Tang J, and Zaidi H 2013, "Four-dimensional image reconstruction strategies in cardiacgated and respiratory-gated PET imaging," PET Clinics 8(1) 51-67.

Reinhardt J M, Ding K, Cao K, Christensen G E, Hoffman E A and Bodas S V 2008, "Registration-based estimates of local lung tissue expansion compared to xenon CT measures of specific ventilation," Med Image Anal. 12(6) 752-63.

Rezaei A, Michel C, Casey M E, and Nuyts J 2016, "Simultaneous reconstruction of the activity image and registration of the CT image in TOF-PET," Physics in Medicine and Biology 61(4) 1852-74.

Rhodes C G and Hughes J M B 1995, "Pulmonary studies using positron emission tomography," European Respiratory Journal 8 1001-17. 
Scherer P M and Chen D L 2016, "Continuing Education on Imaging Pulmonary Inflammation," J Nucl Med. jnumed.115.157438.

Simon B A 2000, "Non-Invasive Imaging of Regional Lung Function using X-Ray Computed Tomography," Journal of Clinical Monitoring and Computing 16(5) 433-42.

Stamm G and Nagel H D 2002, "CT-expo, a novel program for dose evaluation in CT," Rofo 174(12) $1570-6$.

Sun T and Mok G S P 2012, "Techniques for respiration-induced artifacts reductions in thoracic PET/CT," Quant Imaging Med Surg. 2(1) 46-52.

Thielemans K, Asma E, and Manjeshwar R M 2009, "Mass-preserving image registration using free-form deformation fields," IEEE Nuclear Science Symposium and Medical Imaging Conference 2490-2495.

Ue H, Haneishi H, Iwanaga H, and Suga K 2006, "Nonlinear motion correction of respiratory-gated lung SPECT images," IEEE transactions on medical imaging 25(4) 486-95.

Verschakelen J A, Van fraeyenhoven L, Laureys G, Demedts M and Baert A L 1993, "Differences in CT density between dependent and nondependent portions of the lung: influence of lung volume," American Journal of Roentgenology 161(4) 713-17.

Vines D C, Keller H, Hoisak J D and Breen S L 2007, "Quantitative PET comparing gated with nongated acquisitions using a NEMA phantom with respiratory-simulated motion," Journal of nuclear medicine technology 35(4) 246-51.

Wells R G, Ruddy T D, DeKemp R A, DaSilva J N and Beanlands R S 2010, "Single-phase CT aligned to gated PET for respiratory motion correction in cardiac PET/CT," Journal of Nuclear Medicine 51(8) 1182-90.

Win T, Screaton N J, Porter J, Endozo R, Wild D, Kayani I, Dickson J C, Shortman R I, Reubi J C, Ell P J and Groves A M 2012, "Novel positron emission tomography/computed tomography of diffuse parenchymal lung disease combining a labeled somatostatin receptor analogue and 2-deoxy2[18f]fluoro-d-glucose," Mol. Imaging 11(2) 91-8.

Win T, Thomas B A, Lambrou T, Hutton B F, Screaton N J, Porter J C, Maher T M, Endozo R, Shortman R I, Afaq A, Lukey P, Ell P J and Groves A M 2014, "Areas of normal pulmonary parenchyma on HRCT exhibit increased FDG PET signal in IPF patients," Eur. J. Nucl. Med. Mol. Imaging 41(2) 337-42.

Wollmer P, Rhodes C G and Hughes J M 1984, "Regional extravascular density and fractional blood volume of the lung in interstitial disease," Thorax 39 286-93.

Yin Y, Hoffman E and Lin C-L 2009, "Mass preserving nonrigid registration of CT lung images using cubic B-spline," Med. Phys. 36(9) 4213-22. 